\title{
PATOLOGÍAS BUCALES EN CRÁNEOS HUMANOS DEL NORESTE DE PATAGONIA: TENDENCIAS TEMPORALES EN LA DIETA Y ESTADO DE SALUD DE LAS POBLACIONES DEL HOLOCENO-TARDÍO DEL VALLE INFERIOR DEL RÍO NEGRO
}

\author{
LUMILA PAULA MENÉNDEZ"
}

\begin{abstract}
RESUMEN
El objetivo de este trabajo es contribuir, desde la bioarqueología, a la discusión del modelo de intensificación económica y ampliación de la dieta de las poblaciones cazadoras-recolectoras tardías del noreste de Patagonia propuesto por diferentes autores. El presupuesto subyacente es que dicho proceso habría introducido cambios detectables en la distribución intra e interpoblacional de indicadores de salud y función oral. Para evaluar esta proposición, se analizaron las tendencias temporales en las prevalencias de distintos indicadores dentales y periodontales relevantes (caries, abscesos y pérdidas de dientes antemortem, alteraciones periodontales, dislocación de las piezas dentales y labiación del borde alveolar) en una colección formada por tres submuestras diacrónicas de cráneos procedentes del valle inferior del río Negro que cubren los últimos 3.600 años cal. AP. Los resultados obtenidos muestran que las prevalencias de caries, alteraciones periodontales y labiación tienden a incrementarse a través del tiempo, mientras que las de pérdida de dientes antemortem, abscesos y dislocación tienden a decrecer. Tomadas en conjunto, éstas tendencias temporales son consistentes con la hipótesis de aumento en el consumo de recursos vegetales, particularmente de aquellos con potencial cariogénico (v.g. farináceos ricos en hidratos de carbono), con posterioridad al $1.000 \mathrm{AP}$.
\end{abstract}

PALABRAS CLAVES: noreste de Patagonia, cazadores recolectores, salud bucal, sudeste de Pampa.

$$
\begin{aligned}
& \text { ORAL HEALTH IN HUMAN SKULLS FROM NORTH-EAST PATAGONIA: } \\
& \text { TEMPORAL TRENDS IN DIET AND HEALTH STATUS OF HUMAN } \\
& \text { POPULATIONS DURING LATE HOLOCENE IN LOW RIO NEGRO VALLEY }
\end{aligned}
$$

\footnotetext{
ABSTRACT

The aim of this paper is to contribute, from a bioarchaeological perspective, to the discussion about the model of economic intensification and diet broadening of hunter-gatherer populations in northeastern Patagonia previously proposed by different authors. The underlying presuposition is that such process would have detectable effects in the intra-and interpopulational distribution of health and oral function indicators. To test this proposition, we analyzed temporal trends in the prevalence of different dental and 
periodontal relevant indicators (caries, abscesses and loss of antemortem teeth, periodontitis, tilting and lipping) in a collection formed by three diachronic subsamples of skulls from the río Negro valley covering the last 3.600 years $\mathrm{AB} 14 \mathrm{C}$. The results show that the prevalence of caries, periodontal disease and lipping tend to increase over time, while those of antemortem tooth loss, abscesses and tilting decrease. Taken together, these temporal trends are consistent with the hypothesis of increased consumption of plant resources, particularly those with cariogenic potential (i.e. high starches content), after 1.000 AB.

KEYWORDS: northeastern Patagonia, hunter-gatherers, oral health, southeastern Pampa.

\section{INTRODUCCIÓN}

Un conjunto de evidencia sugiere que, durante el Holoceno tardío y con posterioridad a la denominada Anomalía Climática Medieval (ca. 850-1350 años cal. AD; Stine 1994), la población humana del NE de Patagonia habría experimentado un proceso de expansión y dispersión que alcanzó, por lo menos, el Sistema Serrano de Tandilla en el SE de la Región Pampeana (Barrientos 1997, 2001). Barrientos y Perez (2004) propusieron un modelo que da cuenta del mencionado proceso, relacionándolo con cambios en la organización de las sociedades norpatagónicas en diferentes esferas, tales como la demografía, la organización social, política, económica e ideológica, que habrían constituido las principales razones del proceso dispersivo. Desde un punto de vista económico se ha enfatizado, tanto en el NE de Patagonia como en el SE de la Región Pampeana, en la existencia de una ampliación de la dieta y de una intensificación en el uso de ciertos recursos durante el Holoceno tardío, principalmente vegetales (Barrientos 2001; Martínez 2004; Martínez y Gutiérrez 2004; Mazzanti 2006; Politis 2008, entre otros). L'Heureux (1998), demostró la existencia de un aumento estadísticamente significativo en la prevalencia de caries y enfermedades periodontales en muestras tardías del SE de la Región Pampeana -que manifiestan características biológicas y culturales afines a las del NE de Patagonia-, respecto a la de otros momentos del Holoceno. Esta evidencia tendería a apoyar la hipótesis de intensificación económica y ampliación de la dieta en estas poblaciones, manifestados a través del aumento en el procesamiento y consumo de alimentos cariogénicos (i.e. hidratos de carbono de origen vegetal).

En este contexto, un aspecto relevante a investigar es la existencia de tendencias temporales en los valores de prevalencia de caries y alteraciones periodontales -adecuados indicadores de la dieta $y$ de los patrones de salud/enfermedad a escala poblacional- en el NE de Patagonia, mediante el estudio de muestras diacrónicas de restos óseos humanos procedentes de esa región. En este sentido, el objetivo de este trabajo es evaluar la prevalencia de distintos indicadores dentales y periodontales (caries, abscesos, pérdidas de dientes ante-mortem, alteraciones periodontales, dislocación de las piezas dentales y labiación del borde alveolar) en muestras del NE de Patagonia (valle inferior del río Negro) correspondientes a los períodos 3.400-2.900 años cal. AP, 2.800-2.300 años cal. AP y 600-300 años cal AP (Barrientos et al. 2009) y comparar los resultados obtenidos con aquellos producidos por estudios previos llevados a cabo con muestras del Holoceno tardío del SE de la Región Pampeana.

\section{MATERIALES Y MÉTODOS}

La muestra seleccionada para este estudio corresponde a la colección Moreno (Moreno 1874), depositada en la División Antropología de la Facultad de Ciencias Naturales y Museo de la Universidad Nacional de La Plata (Lehmann-Nitsche 1910). Esta muestra, procedente del valle inferior del río Negro (Figura 1), ha sido escogida debido a la profundidad temporal que presenta, cuyo rango cronológico se extiende desde inicios del Holoceno tardío (ca. 3.600 años cal. AP) hasta momentos históricos tempranos (ca. 300 años cal AP) (Bernal et al. 2008; Barrientos et al. 2009). La misma está compuesta por 221 cráneos o calvarias, que corresponden en su mayoría (212/221) a individuos adultos, de los cuales 100 son femeninos, 98 masculinos y 23 no determinados. La determinación del sexo y estimación de edad fueron realizadas siguiendo los criterios resumidos por Buikstra y Ubelaker (1994). La determinación 


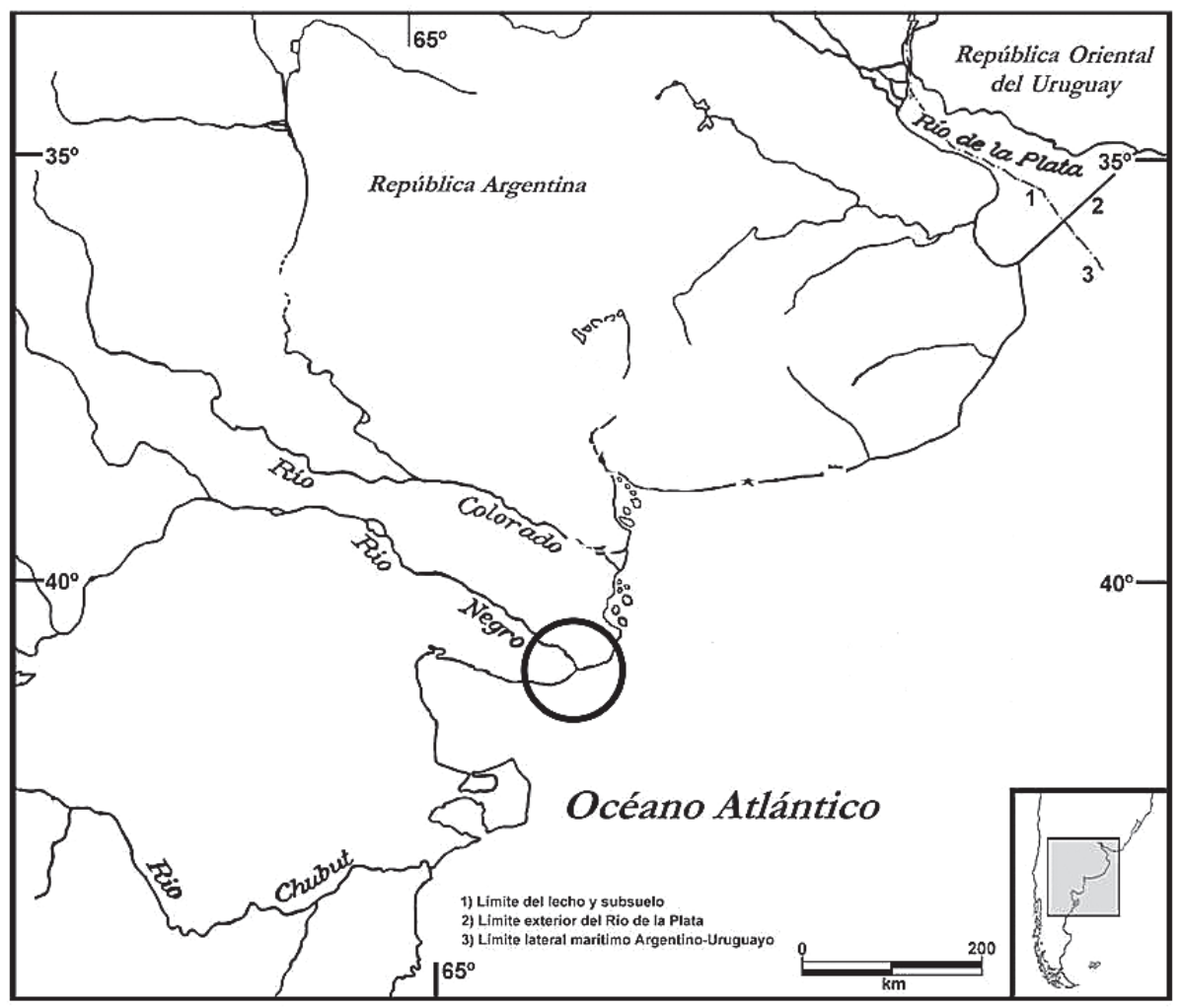

Fig. 1. Procedencia geográfica de la muestra analizada.

del sexo fue realizada a partir de la evaluación de los siguientes rasgos morfológicos: glabella, margen supraorbitario, proceso mastoideo, arcos superciliares, cresta supramastoidea, y el aspecto general de la mandíbula, cuando ésta estaba presente. La estimación de la edad fue realizada considerando el cierre de las suturas, específicamente aquellas correspondientes al sistema anterior-lateral definido por Meindl y Lovejoy (1985), que es considerado como el criterio más confiable utilizando este tipo de indicador etáreo.

La muestra fue subdividida en submuestras diacrónicas (Tabla 1). Para este fin, se tuvieron en cuenta las deformaciones craneanas artificiales (circular-pseudocircular, plano-frontal o tabular oblicua y tabular erecta planolámbdica) que, en el norte de Patagonia y en la Región Pampeana, constituyen un eficiente rasgo de discriminación temporal, con anclajes en cronologías absolutas en las provincias de Chubut, Río Negro, La Pampa, Mendoza y Buenos Aires (Baffi y Berón 1992, Barrientos 2001; Barrientos y Perez 2002, Barrientos et al. 2009; Béguelin et al.
2006, Bernal et al. 2008, Berón y Baffi 2003, Berón y Luna 2009, Bórmida 1953/1954; Gómez Otero y Dahinten 1997/1998). La deformación craneana circular y/o pseudocircular presente en el Valle de río Negro, ha sido datada radiocarbonicamente en el sudeste de la Región Pampeana y en Pampa Seca con antigüedades superiores a los 2.900 años AP. Recientemente, Bernal et al. (2008) y Barrientos et al. (2009) han obtenido dataciones de cráneos procedentes del valle del Río Negro que indican una cronología local para este tipo de deformación situada entre por lo menos 3.400 y 2.900 años cal. AP. Las deformaciones tabular erecta plano-frontal y tabular oblicua, en general coetáneas, han sido datadas en el valle de Chubut, en la costa del golfo Nuevo, en la costa NO del Golfo de San Matías y en SE de la región Pampeana con antigüedades situadas entre 2.800 y 1.800 años AP (Gómez Otero y Dahinten 1997/1998; Madrid y Barrientos 2000; Favier Dubois et al. 2009; Politis 1984; Politis et al. 2009). Los fechados obtenidos por Bernal et al. (2008) y Barrientos et al. (2009) para estos tipos de defor- 
Tabla 1. Cronología de los períodos según deformación craneana.

\begin{tabular}{|c|c|c|}
\hline Período & Deformación craneana & Cronología \\
\hline I & Circular/Pseudocircular & $3.400-2.900$ años cal. AP \\
\hline II & Plano-frontal & $2.800-2.300$ años cal. AP \\
\hline III & Plano-lámbdica & $600-300$ años cal. AP \\
\hline
\end{tabular}

maciones en cráneos del valle inferior del río Negro, se distribuyen dentro del lapso 2.800-2.300 años cal. AP. Finalmente, en relación con la deformación tabular erecta, particularmente de su variante plano lámbdica -la de más amplia distribución y frecuencia en Pampa y Patagonia (Barrientos 2001; Bórmida 1953/1954)-, trabajos previos indican que en estas dos regiones esta deformación ocurre entre 1900 años cal. AP y momentos históricos (Béguelin et al. 2006; Gómez Otero y Dahinten 1997/1998; Berón y Luna 2009; Martínez 2004; Martínez et al. 2007). Bernal et al. (2008) y Barrientos et al. (2009) obtuvieron para muestras del valle inferior del río Negro con este tipo de deformación fechas situadas dentro del lapso 600-300 años cal AP. Los cráneos no deformados, que constituyen el 51,8\% de la muestra total disponible no fueron incluidos en este estudio, ya que se distribuyen a todo lo largo de la secuencia temporal del valle inferior del río Negro, desde aproximadamente 3.600 años cal. AP (Barrientos et al. 2009).

El conjunto de indicadores fue analizado mediante observación escópica con una lupa de pié de luz fría de 3X siguiendo, en gran medida, la metodología propuesta por L'Heureux (1998, 2002), quien llevó a cabo investigaciones sobre indicadores de salud bucal en el SE de la Región Pampeana, lo que ha permitido realizar la comparación de los resultados. Los indicadores considerados en el presente estudio son: a) caries, b) abscesos, c) pérdidas de dientes antemortem (PAM), d) alteraciones periodontales, e) dislocación de las piezas dentales y f) labiación del borde alveolar. A continuación, se realizará una breve descripción de la metodología utilizada para el análisis de cada variable.

Las caries, son entendidas como el resultado de un proceso caracterizado por la desmineralización focal de los tejidos duros del diente (Hillson 2008, Larsen 1987, Larsen et al. 1991, LenanderLumikari y Loimaranta 2000, Mc Hug 1970). La correlación negativa entre los elevados grados de desgaste (característica de los grupos cazadoresrecolectores) y la baja prevalencia de caries, sobre todo de tipo coronal-oclusal, ha sido largamente documentada, como así también la correlación positiva existente entre las economías agrícolas o de transición (caracterizadas por el consumo de alimentos blandos, pegajosos, con azúcares) y la alta prevalencia de caries, tanto coronales como radiculares y tanto oclusales como interproximales (Hillson 1990, 2008, Larsen et al. 1991, Walker y Erlandson 1986, entre otros). Las caries se relevaron utilizando la propuesta de Buikstra y Ubelaker (1994), la cual categoriza numéricamente a las caries según su localización a partir de la inspección escópica de cada pieza dental. Asimismo, se calculó la Tasa Corregida de Caries (Lukacs, 1995), dado que la muestra presenta un gran número de dientes perdidos ante-mortem. Los abscesos son el resultado de una enfermedad pulpo-alveolar localizada, producto de la exposición, contaminación bacteriana y muerte del tejido pulpar (Ogden 2008b, Oxenham et al. 2006). Siguiendo a Buikstra y Ubelaker (1994), se discriminaron los diversos tipos de abscesos según su localización (i.e. labial y lingual). Las pérdidas de dientes antemortem (PAM) son aquellas pérdidas de piezas dentales que ocurren durante el transcurso de la vida de un individuo, relacionadas con presiones masticatorias y con la presencia de patologías orales (Lukacs 2006). El relevamiento de este indicador se realizó de forma macroscópica en todos los alvéolos, de acuerdo con el sistema propuesto por Buikstra y Ubelaker (1994), que adjudica un código numérico según la localización. La enfermedad periodontal es entendida en el presente trabajo como una degeneración de los tejidos de soporte del diente, es decir de la gingiva, del cemento, de los ligamentos periodontales y del hueso alveolar (Clarke y Hirsch 1991a, Hildebolt y Molnar 1991, Keenleyside 2003). La modificación de la morfología normal del hueso alveolar se examinó y registró teniendo en cuenta los siguientes criterios morfológicos (Ogden 2008a): 1) pérdida de la forma de la cresta alveolar, 2) porosidad del hueso cortical, 3) osteoporosis en el septum interdental, 4) presencia de inflamación ósea y de 
adelgazamiento o pérdida de hueso alveolar. La dislocación o "tilting" refiere a la inclinación de la corona dental hacia el lado lingual y del o de los ápices de la raíz, en dirección bucal (Clarke y Hirsch 1991b, Molnar 2008). Se evaluó en la totalidad de las piezas dentales presentes la presencia/ausencia de este rasgo. La labiación o "lipping" del margen de la cresta alveolar es el crecimiento excesivo del hueso más allá de los márgenes de la cresta alveolar como producto de una sobreexigencia en el funcionamiento del aparato masticatorio (Buikstra y Ubelaker 1994, Larsen 2006). Para examinar este indicador, se observaron todos los bordes bucales de los alvéolos de la dentición posterior.

Se considera que la labiación y la dislocación son respuestas adaptativas que ocurren como procesos compensatorios, cuando existen tasas de degaste moderadas a severas. Por lo tanto, si bien en el presente trabajo no se han relevado ni los tipos ni los grados de desgaste dental, se considera que su presencia está directamente relacionada con estas variables y son, por lo tanto, básicamente independientes de la enfermedad periodontal.

Para realizar el relevamiento de los indicadores considerados, se observaron un total 2996 alveolos [Período I (3400-2900 años cal. AP) n= 472; Período II (2800-2300 años cal. AP) $n=$ 232; Período III (600-300 años cal AP) $n=712$; Cronología Indeterminada $n=1580)$ ], en los cuales se encontraban presentes un total de 1317 dientes (Período I $n=221$; Período II $n=110$; Período III $\mathrm{n}=297$; Cronología Indeterminada $\mathrm{n}=689$ ).

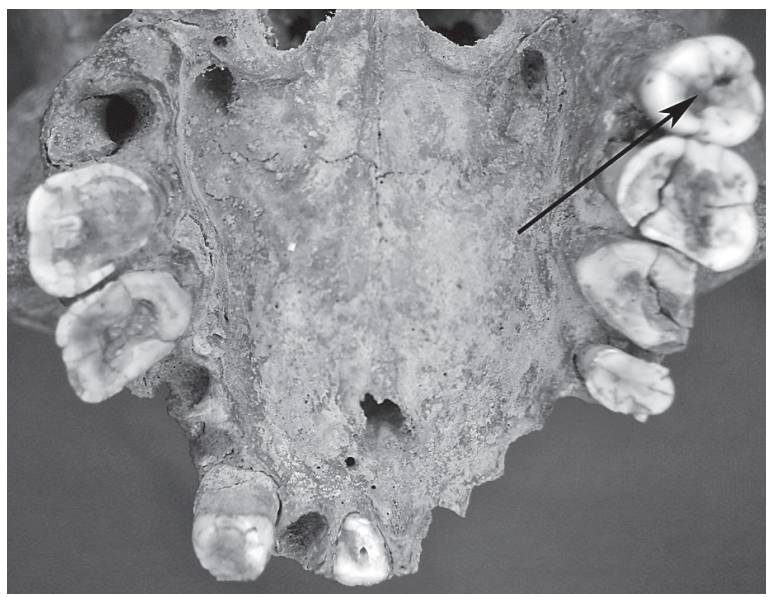

Fig. 2. Caries presente en superficie oclusal del M3 del maxilar derecho.
Los datos fueron analizados mediante el uso de estadística descriptiva e inferencial, a través de la puesta a prueba de hipótesis de nulidad, i.e. aquellas que afirman la no existencia de diferencias significativas entre muestras. Para esto, se utilizó el programa STATISTICA (versión 7). Se aplicaron los test no paramétricos $\chi^{2}$ y exacto de Fisher, según correspondiera en cada caso, con el fin de conocer la existencia de diferencias significativas entre períodos en los valores de prevalencia obtenidos para cada indicador. En todos los casos, el nivel de significación alfa se fijó en 0,05.

\section{RESULTADOS}

Las caries, se encuentran localizadas mayoritariamente en la superficie oclusal $(n=25)$ y, en segundo lugar, en las superficies de contacto interproximal $(n=5)$ (Figura 2). Asimismo, se presentan con mayor frecuencia en los dientes posteriores. En general, cuando este indicador ha estado presente, se ha detectado una caries por diente, aunque hay casos en los que aparecen hasta dos caries en una misma pieza dental. La tasa total de caries observada es 0.01 mientras que el Factor de Corrección de Caries sensu Lukacs (1995) duplicó este valor resultando 0.02. Las Tasas Corregidas de Caries se incrementan en el tiempo con los siguientes valores: $\mathrm{PI}=0.00, \mathrm{PII}=0.02$ y PIII=0.05. Los abscesos, que son mayoritariamente de tipo bucal periapical, presentan frecuencias más altas en los dos primeros molares, aunque también se han detectado en otros dientes (Figura 3). Se ha

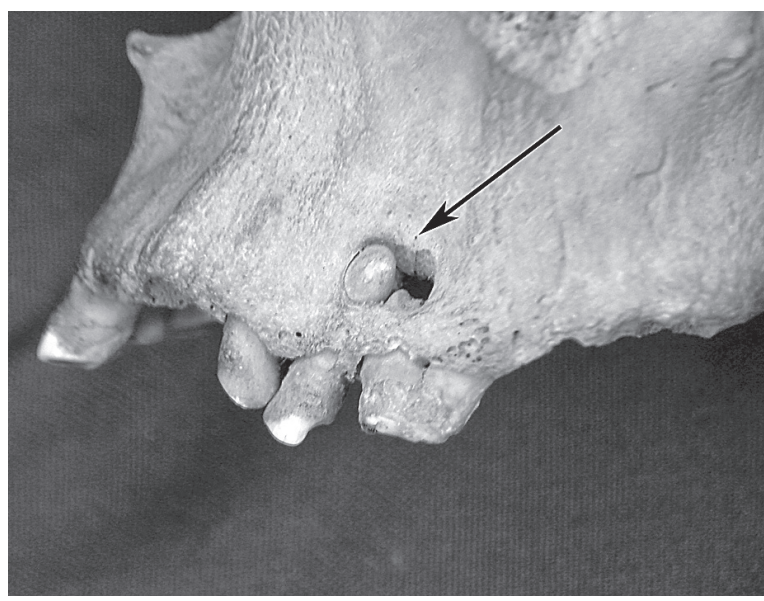

Fig 3. Absceso periapical presente en Pm2-M1 de maxilar izquierdo. 


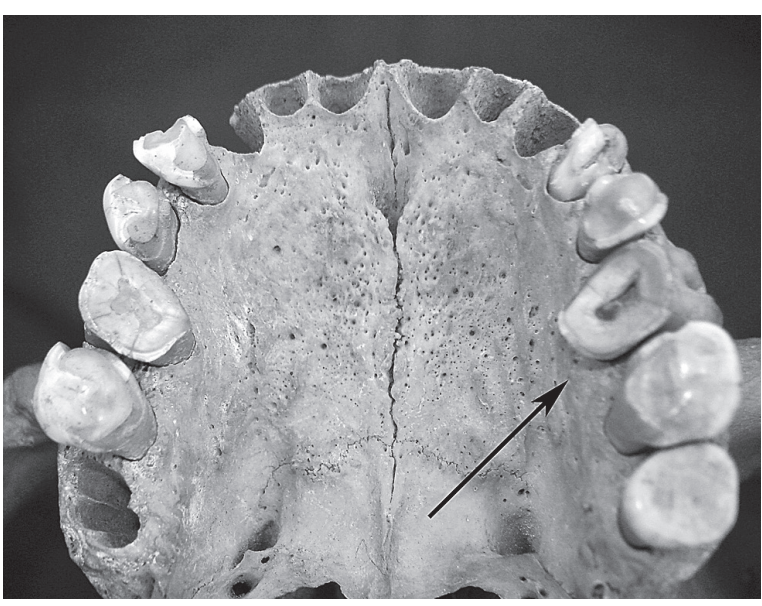

Fig. 4. Dislocación presente en M1 de maxilar izquierdo.

registrado una pequeña cantidad de abscesos en la cara lingual de diferentes tipos de dientes, existiendo algunos casos en los que se presentaron, en una misma pieza, un absceso lingual y otro bucal. La dislocación se encontró mayoritariamente presente en el primer molar, aunque hay casos en los que se presentó en el segundo molar (Figura 4). La labiación se encontró en todos las casos a la altura de los molares mandibulares (Figura 5). Las PAM están presentes en toda la arcada alveolar, aunque mayoritariamente fueron los dientes posteriores los que presentaron una mayor tendencia a ser perdidos in vivo (Figura 6). Las alteraciones periodontales, si bien se observa en todos los dientes, es más acentuada en la región de los dientes posteriores

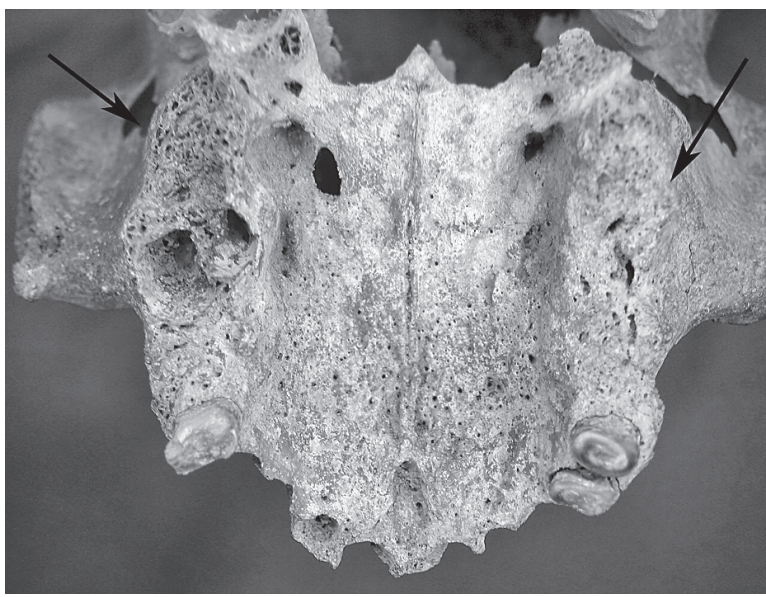

Fig. 6. Múltiples pérdidas de dientes antemortem presentes en maxilar.

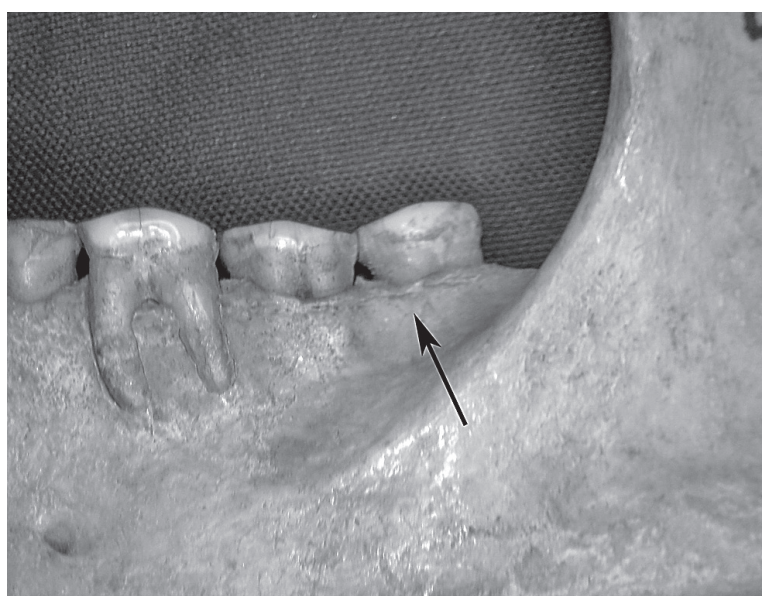

Fig. 5. Labiación presente a la altura de M2-M3 de mandíbula derecha.

(Figura 7). Si bien se encuentra presente en las tres submuestras, en la más tardía (P III), se presenta en algunos casos con un grado de severidad alto (Grado 4; Ogden, 2008a).

Sobre los valores de prevalencia obtenidos (Tabla 2), se aplicaron las pruebas estadísticas orientadas a detectar la presencia de diferencias significativas entre los períodos considerados. Se encontraron diferencias estadísticamente significativas cuando se compararon las prevalencias de caries, dislocación dental, PAM, labiación, y abscesos entre las submuestras P I/P III, y abscesos entre P II/P III. No existen diferencias significativas en los valores de las alteraciones periodontales, presentando valores similares en las tres submuestras, con una

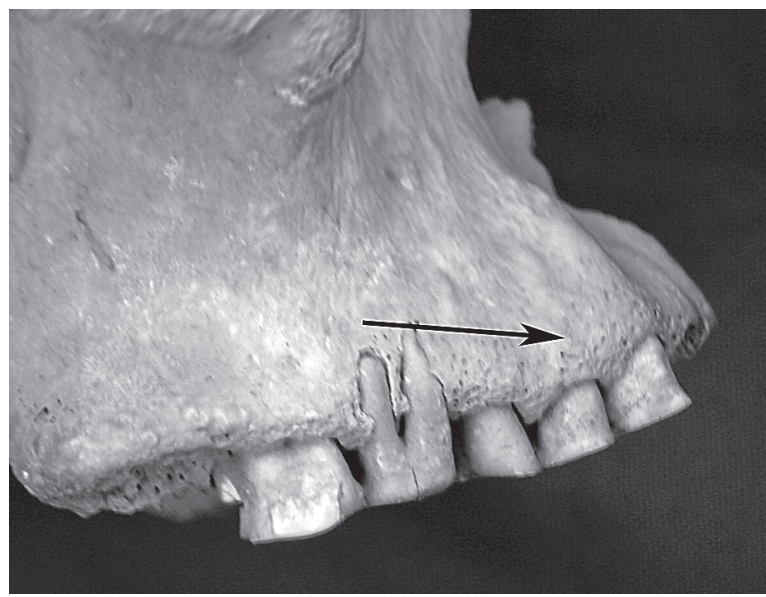

Fig. 7. Alteraciones periodontales presentes en maxilar derecho. 
Tabla 2. Prevalencias de cada indicador por período.

\begin{tabular}{|c|c|c|c|c|c|c|}
\hline & \multicolumn{2}{|c|}{ Período I } & \multicolumn{2}{c|}{ Período II } & \multicolumn{2}{c|}{ Período III } \\
\hline & $\mathbf{N}$ & Prevalencia & N & Prevalencia & N & Prevalencia \\
\hline Caries & 221 & 00,90 & 110 & 02,72 & 297 & 06,06 \\
\hline Dislocación & 221 & 11,76 & 110 & 06,36 & 297 & 05,38 \\
\hline PAM & 422 & 09,00 & 232 & 04,74 & 712 & 03,37 \\
\hline Labiación & 48 & 00,00 & 32 & 09,37 & 160 & 11,87 \\
\hline Abscesos & 422 & 08,29 & 232 & 09,05 & 712 & 03,79 \\
\hline Alt.periodontales & 422 & 15,63 & 232 & 17,83 & 712 & 18,53 \\
\hline
\end{tabular}

leve tendencia al aumento en la submuestra del $\mathrm{P}$ III. Respecto de las caries, se encontraron diferencias significativas entre P I y P III ( $F=7,74 ; p \leq 0,01)$, presentando valores más altos en el último período. Respecto de los abscesos, se encontraron diferencias estadísticamente significativas entre P I y P III $\left(\chi^{2}=9,54 ; \mathrm{p} \leq 0.01\right)$, así como también entre P II y P III $\left(\chi^{2}=8,97, p \leq 0,01\right)$, siendo las prevalencias del $P$ III las menores para ambos casos. Para labiación, las diferencias fueron significativas para P I/P III $(F=4,93 ; p \leq 0,01)$, siendo menores las frecuencias en la primera. Respecto a dislocación, se encontraron diferencias significativas entre P I y P IIII $\left(\chi^{2}=6,09\right.$; $p \leq 0.05$ ), siendo las prevalencias menores en el último período. Finalmente, para las PAM se encontraron diferencias estadísticamente significativas entre P I y PIII $\left(\chi^{2}=15,20 ; p \leq 0.01\right)$, siendo mayores las frecuencias en el primer período (Tabla 3).

En cuanto a la comparación de estos resultados con aquellos obtenidos por L'Heureux (1998, 2000, 2002) para muestras del SE de la Región Pampeana, existen muchas similitudes en las tendencias que presentan los indicadores considerados: caries, alteraciones periodontales y labiación se incrementan, mientras que los abscesos, las dislocaciones y las PAM decrecen. Sin embargo, en el SE de la Región Pampeana, las únicas diferencias significativas entre los períodos se presentaron en los valores de prevalencia de caries y labiación.

Si comparamos las similitudes de las prevalencias obtenidas para ambas regiones, caries y labiación se incrementan significativamente, mientras abscesos, dislocación y PAM decrecen, aunque sólo en el NE de Patagonia las diferencias son estadísticamente significativas.

Dado que la muestra del Holoceno Tardío Final de la Región Pampeana es aquella para la cual se ha postulado (Barrientos 1997, Barrientos y Perez 2004) las mayores afinidades, tanto biológicas como culturales, con muestras del NE de Patagonia (las correspondientes al P III) es relevante establecer comparaciones entre estas muestras. Por esto, se procedió a comparar los valores de la muestra de Holoceno tardío del SE de la Región Pampeana con el total de los valores obtenidos en las muestras analizadas en el presente estudio. A primera vista, los valores de caries, abscesos y alteraciones periodontales son similares en ambas muestras, mientras que los valores de PAM, dislocación y labiación difieren. De esta forma se procedió a realizar distintos test de $\chi^{2}$ para comparar las frecuencias de valores de los indicadores para ambas regiones. Se encontraron diferencias significativas en a) dislocación, cuyas prevalencia en el SE de Pampa es 1,94 y en el valle del río Negro 7,89; y b) PAM, cuya prevalencia en el SE de Pampa es 1,71 y en el valle del río Negro 7,60. No se encontraron diferencias significativas en: a) caries ( $\mathrm{Pampa}=3,44$; Patagonia $=3,11$ ), b) labiación (Pampa $=19,79$; Patagonia $=6,48)$, c) abscesos (Pampa=6,87; Patagonia $=6,27)$ y d) alteraciones periodontales (Pampa=16,66; Patagonia=14,76).

Tabla 3. Valores resultantes de las pruebas de significación $\chi^{2}$ y Test Exacto de Fisher.

\begin{tabular}{|c|c|c|c|}
\hline & PI/PII & PII/III & PI/PIII \\
\hline Dislocación & 01,82 & 00,02 & $06,09^{*}$ \\
\hline PAM & 03,33 & 00,58 & $15,20^{* *}$ \\
\hline Abscesos & 00,03 & $08,97^{* *}$ & $09,54^{* *}$ \\
\hline Alt. periodontales & 00,71 & 00,02 & 00,76 \\
\hline Caries $^{1}$ & 00,64 & 01,21 & $07,74^{* *}$ \\
\hline Labiacion $^{1}$ & 02,44 & 00,01 & $04,92^{* *}$ \\
\hline
\end{tabular}

$\left({ }^{*}=\mathrm{p} \leq 0.05 ;{ }^{* *}=\mathrm{p} \leq 0.01 ;{ }^{1}=\right.$ Valores según Test Exacto de Fisher). 


\section{DISCUSIÓN Y CONSIDERACIONES FINALES}

A partir de la prevalencia de los indicadores en las muestras analizadas, se observa que algunos se incrementan en el tiempo (caries, alteraciones periodontales, labiación de la cresta alveolar), mientras que otros disminuyen (dislocación dental, PAM, abscesos, Fig. 8).

A partir de éstos resultados, se puede plantear un cuadro donde un grupo de variables decrece conjuntamente: abscesos, pérdidas ante-mortem y dislocación. Es decir, estos indicadores tienen valores altos en momentos más tempranos y disminuyen su frecuencia o severidad con el tiempo. Clarke y Hirsch (1991b) han planteado un modelo en el cual la dislocación, altas tasas de desgaste y abscesos se encuentran correlacionados. Cuando existen altas tasas de desgaste, se produce progresivamente la erupción continua de los dientes para mantener constante la altura facial y, consecuentemente, estable la función mandibular, ya que una brusca modificación del ángulo entre la rama ascendente de la mandíbula y la base del cráneo afecta a la articulación cóndilo-temporal y a la superficie oclusal de los dientes, alterando el patrón de masticación (Whittaker et al. 1990). A partir de la erupción continua secundaria a la existencia de altas tasas de desgaste dental, los dientes -particularmente los molares- van perdiendo superficie de sujeción lo cual, sumado a las fuerzas de la masticación, llevan a que los dientes disloquen a la corona lingualmente y a las raíces bucalmente. Cuando el diente se ha dislocado a tal punto que las raíces pasan a través de los tejidos gingivales, los canales de las raíces infectados se separan del ambiente interno mientras el diente sigue funcionando. La consecuencia más típica de la combinación entre desgaste dental severo y formación de abscesos dentales, es la pérdida del diente (Ogden 2008). La dislocación y la labiación del borde alveolar son, pues, procesos fisiológicos compensatorios de tasas de desgaste severas. La dislocación representa la mejor de las posibles adaptaciones a las consecuencias que llevan a la pérdida del sostén del diente, mientras que la labiación del borde alveolar es una respuesta ósea adaptativa a una sobreexigencia en el funcionamiento del aparato masticatorio. Como vimos, la combinación de estos factores impide la ocurrencia de modificaciones del ángulo mandibular y la generación de atrofias de la articulación temporo-mandibular, situación que favorecería a aquellas poblaciones con dietas que incluyen grandes cantidades de abrasivos -sea por la naturaleza de los alimentos o por las técnicas de almacenaje y preparación de los mismos-, que presentan tendencia a experimentar desgaste dental severo, al posibilitar una acción masticatoria satisfactoria por un tiempo más prolongado.

Si bien en esta investigación no se ha relevado la tasa de degaste dental, estudios previos sobre la misma muestra (Bernal et al. 2007) indican que la submuestra del P I presenta las mayores tasas de desgaste dental en el valle inferior del río Negro. En esta región, las caries, se incrementan en el tiempo. El depósito de placa es un factor común que predispone la presencia de caries y alteraciones periodontales, entre otras patologías, y el mismo

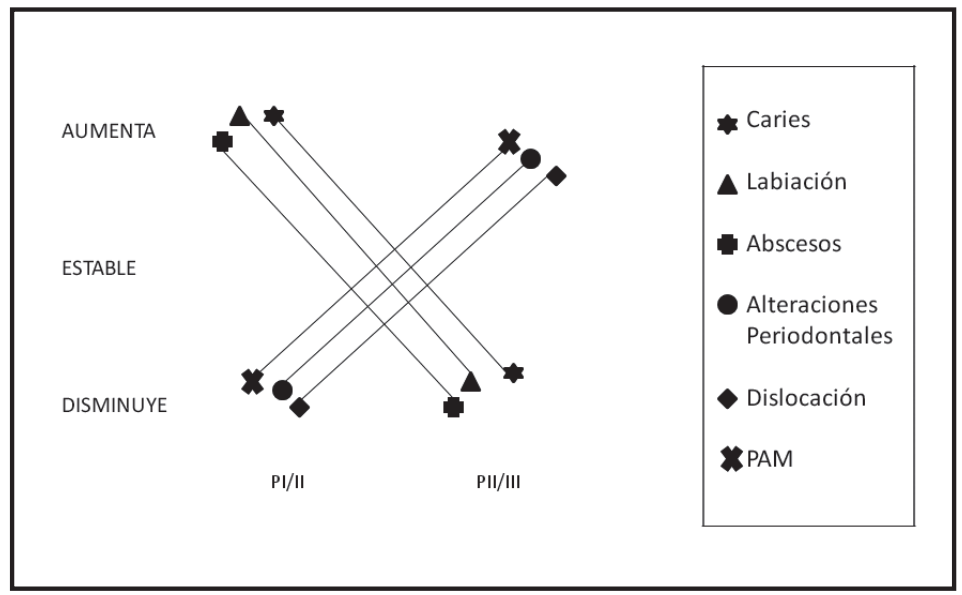

Fig. 8. Tendencias temporales de los indicadores analizados a partir de valores $\mathrm{p}$. 
es originado por una dieta alta en carbohidratos así como también otros factores como pueden ser crowding, maloclusión e higiene oral pobre (Hillson 1986, Ogden 2008a). La medida del tamaño de la caries y su localización proveen información acerca de su severidad: en grupos cazadores-recolectores se encuentran restringidas al cuello de la dentición posterior, mientras en agricultores se encuentran presentes en múltiples lugares. En el presente estudio existe una alta frecuencia de caries oclusales $y$, en segundo término, interproximales y de cuello. Las caries oclusales aumentan en relación con el consumo de carbohidratos (Hillson 1986) y la disminución de la tasa de desgaste dental, que favorece su formación (ver discusión en L'Heureux 1998). La prevalencia general de caries obtenida para la muestra total y para los diferentes periodos, se adecua a los promedios de prevalencias de patologías cariosas sugeridos por Turner (1979) y Larsen (1987) para los grupos cazadores-recolectores (0,0 \% - 7,8 \%). Dado que las caries incrementan su frecuencia a través del tiempo, alcanzando prevalencias $(6,6 \%)$ cercanas al límite propuesto para cazadores-recolectores, esta tendencia sugiere un aumento en el consumo de productos cariogénicos en el Holoceno tardío final. Esto es compatible con el cambio inferido en esta región en las estrategias económicas orientadas a un mayor consumo de recursos vegetales a partir de aproximadamente el 1.000 AP.

Existen numerosas evidencias arqueológicas que permiten correlacionar altos niveles de consumo de carbohidratos con altas tasas de enfermedad periodontal. De hecho, los prerrequisitos para la formación de una caries son en términos generales los mismos que para la enfermedad periodontal: presencia de placa y el consumo de carbohidratos en la dieta. Ambas patologías son el producto de procesos patológicos relacionados con el consumo alto de carbohidratos en la dieta (Hillson 1986, Larsen 1997).

En suma, los resultados obtenidos en el presente estudio son concordantes con otros estudios regionales realizados con muestras similares a las aquí analizadas (L'Heureux 1998, 2002). En conjunto, permiten sostener que las poblaciones del NE de Patagonia y del SE de la Región Pampeana durante el Holoceno tardío fueron incorporando progresivamente a su dieta elementos cariogénicos, tal como plantean otros investigadores para la re- gión, donde recursos como frutos de los vegetales del espinal (algarrobo, chañar, caldén) así como gramíneas silvestres, habrían tenido una importancia mayor a partir de aproximadamente el año 1000 AP (Barrientos, 1997; Barrientos y Perez, 2004; Bernal et al. 2007; Martinez, 1999; Gómez Otero, 2007; Menéndez et al., 2009).

\section{AGRADECIMIENTOS}

Al Dr. Héctor M. Pucciarelli, y personal de la División Antropología de la Facultad de Ciencias Naturales y Museo de la Universidad Nacional de La Plata, por permitirme el acceso a las colecciones a su cargo. A Florencia Gordón por los valiosos comentarios y sugerencias durante las distintas instancias del periodo de la beca que dio origen a este trabajo. A Gustavo Barrientos por su lectura y comentarios. A Federico Lotto y Virginia Cobos, por el aporte que me brindaron las interesantes discusiones con ellos mantenidas. A Valeria Bernal, Laura Ciampagna y Jimena Barbeito por sus críticas y sugerencias.

\section{BIBILIOGRAFÍA}

BAFFI, I. y M. BERÓN. 1992. Los restos óseos humanos de Tapera Moreira (La Pampa) y la deformación artificial en la región Pampeana. Análisis tentativo. Palimpsesto 1: 25-36.

BARRIENTOS, G. 1997. Nutrición y Dieta de las Poblaciones Aborígenes Prehispánicas del Sudeste de la Región Pampeana. Facultad de Ciencias Naturales y Museo, UNLP. Tesis doctoral inédita.

BARRIENTOS, G. 2001. Una aproximación bioarqueológica al estudio del poblamiento prehispánico tardío del sudeste de la Región Pampeana. Intersecciones 2: 3-18.

BARRIENTOS, G. y S. I. PEREZ. 2002. La dinámica del poblamiento humano del Sudeste de la Región Pampeana durante el Holoceno. Intersecciones en Antropología 3: $41-54$.

BARRIENTOS, G. y S. I. PEREZ. 2004. La expansión y dispersión de poblaciones del norte de Patagonia durante el Holoceno tardío: evidencia arqueológica y modelo explicativo. En Contra Viento y Marea. Arqueología de Patagonia, editado por T. Civalero, P. Fernández y G. Guraieb, pp. 179-195. Instituto Nacional de Antropología y Pensamiento Latinoamericano y Sociedad Argentina de Antropología, Buenos Aires. 
BARRIENTOS, G., M. BÉGUELIN y F. GORDÓN. 2009. Tendencias cronológicas en el registro bioarqueológico del noreste de Patagonia. Resúmenes de las IX Jornadas de Antropologia Biológica, realizadas los días 20-23 de octubre en Puerto Madryn, Argentina.pp-96.

BÉGUELIN, M., V.BERNAL, M. DEL PAPA, P. NOVELLINO y G. BARRIENTOS. 2006. El poblamiento humano tardío del sur de Mendoza y su relación con el norte de Patagonia: una discusión bioarqueológica. En $\mathrm{Ar}$ queología y Ambiente de Áreas Naturales Protegidas, editado por V. Duran y V. Cortegoso, pp. 11-32. Anales de Arqueología y Etnología, volumen especial № 61. Universidad Nacional de Cuyo, Facultad de Filosofía y Letras, Mendoza.

BERNAL, V., P. NOVELLINO, P. GONZALEZ, y S. I. PEREZ. 2007. Role of wild plant foods among Late Holocene hunter-gatherers from Central and North Patagonia (South America): an approach from dental evidence. American Journal of Physical Anthropology 133: 1047-1059.

BERNAL, V., P. N. GONZÁLEZ, S. I. PÉREZ y H. M. PUCCIARELLI. 2008. Entierros humanos del noreste de Patagonia: nuevos fechados radiocarbónicos. Magallania 36 (2): 125-134.

BERÓN, M. e I. BAFFI. 2003. Procesos de cambio cultural en los cazadores-recolectores de la provincia de La Pampa, Argentina. Intersecciones en Antropología 4: 29-43.

BERÓN, M. y L. LUNA. 2008. Distribución espacial y cronológica de la deformación craneana tabular erecta en Pampa y Norpatagonia. En Libro de Resúmenes de las VII Jornadas de Arqueología de la Patagonia, pp. 45, realizadas durante los días 21-25 de Abril en el CADIC, Ciudad de Ushuaia, Tierra del fuego.

BERÓN, M. y L. LUNA. 2009. Distribución espacial y cronológica de la deformación craneana tabular erecta en Pampa y Norpatagonia. En Arqueología de la Patagonia. Una Mirada desde el Último Confín, compilado por M. Salemme, F. Santiago, M. Álvarez, E. Piana, M. Vázquez y M. E. Mansur, pp. 561-575. Editorial Utopías, Ushuaia.

BÓRMIDA, M. 1953/1954. Los antiguos Patagones. Estudio de craneología. Runa 6: 55-96.

BUIKSTRA, J. y D. UBELAKER. 1994. Standards for Data Collection from Human Skeletal Remains. Arkansas Archaeological Survey Research Series 44, Fayetteville

CLARKE, N. G. y R. S. HIRSCH. 1991a. Physiological, pulpal, and periodontal factors influencing alveolar bone. En Advances in Dental Anthropology, editado por M. Kelly, y C. Larsen, pp. 241-266. Willey-Liss, Nueva York.
CLARKE, N. G. y R. S. HIRSCH. 1991b. Tooth dislocation: The relationship with tooth wear and dental abscess. American Journal of Physical Anthropology 85: 293-298.

FAVIER DUBOIS, C. M., F. BORELLA y R. H. TYKOT. 2009. Explorando tendencias en el uso humano del espacio y los recursos en el litoral rionegrino (Argentina) durante el Holoceno medio y tardío. En Arqueología de la Patagonia. Una Mirada desde el Último Confín, compilado por M. Salemme, F. Santiago, M. Álvarez, E. Piana, M. Vázquez y M. E. Mansur, pp. 985-997. Editorial Utopías, Ushuaia.

GÓMEZ OTERO, J. y S. DAHINTEN. 1997/1998. Costumbres funerarias y esqueletos humanos: variabilidad y poblamiento en la costa nordeste de la provincia de Chubut (Patagonia Argentina). Relaciones de la Sociedad Argentina de Antropología (N. S.) 22-23:101-124.

HILDEBOLT, C. F. y S. MOLNAR. 1991. Measurement and description of periodontal disease in anthropological studies. En Advances in Dental Anthropology, editado por M. Kelly y C. Larsen, pp. 225-240. Willey-Liss, Nueva York. HILLSON, S. 1986. Teeth. Cambridge University Press.

HILLSON, S. 1990. Theet. Cambridge University Press, Cambridge.

HILLSON, S. 2008. The current state of dental decay. En Technique and Application in Dental Anthropology, editado por J. D. Irish y G. C. Nelson, pp. 111-135. Cambridge University Press, Cambridge.

KEENLEYSIDE, A. 2003. Changing patterns of health and disease among the aleuts. Artic Anthropology 40: 48-69.

LARSEN, C. 1987. Bioarchaeological interpretation of subsistence economy and behavior frum human skeletal remains. Advances in Archaological Method and Theory 10: 339-445.

LARSEN, C.S. 1997. Bioarchaeology. Interpreting behavior from the human skeleton. Cambridge University Press.

LARSEN, C. 2006. The agricultural revolution as environmental catastrophe: Implications for health and lifestyle in the Holocene. Quaternary International 150:12-20.

LARSEN, C., R. SHAVIT, y M. C. GRIFFIN. 1991. Dental caries evidence for dietary change: An archaeological context. En Advances in Dental Anthropology, editado por M. Kelly y C. Larsen, pp. 179-202. Willey-Liss, Nueva York.

L' HEUREUX, G. L. 1998. Biología Oral de las Poblaciones Prehispánicas del Sudeste de La Región Pampeana, Provincia de Buenos Aires. Tesina de Licenciatura en Antropología (Orientación Arqueología). Escuela de Antropología, Facultad de Humanidades y Artes, Universidad Nacional de Rosario. 
L' HEUREUX, G. L. 2000. Estudio comparativo de indicadores de adecuación fisiológica y salud bucal en muestras de restos humanos del Sudeste de la Región Pampeana. Relaciones de la Sociedad Argentina de Antropología (N. S.) 25: 51-73.

L' HEUREUX, G. L. 2002. Inferencias paleodietarias a partir del análisis de los patrones de desgaste dental y frecuencia de caries en muestras de restos humanos del holoceno del sudeste de la región pampeana. En: Del mar a los salitrales. Diez mil años de historia pampeana en el umbral del tercer milenio, editado por Mazzanti, D., M. Berón y F. Oliva. Universidad Nacional de Mar del Plata.

LENANDER-LUMIKARI, M., y V. LOIMARANTA. 2000. Saliva and dental caries. Advances in Dental Research 14:40-47.

LUKACS, J. R. 1995. The 'Caries Correction Factor': a New Method of Calibrating Dental Caries Rates to Compensate for Antemortem Loss of Teeth. International Journal of Osteoarchaeology 5: 151-156.

LUKACS, J. R. 2006. Dental trauma and antemortem tooth loss in prehistoric Canary Islanders: prevalence and contributing factors.International Journal of Osteoarchaeology 17:157-173.

MADRID, P. y G. BARRIENTOS. 2000. La estructura del registro arqueológico en el sitio Laguna Tres Reyes 1(Provincia de Buenos Aires): nuevos datos para la interpretación del poblamiento humano del Sudeste de la Región Pampeana a inicios del Holoceno tardío. Relaciones de la Sociedad Argentina de Antropología (N.S.) 25: 179-206.

MARTÍNEZ, G. 1999. Tecnología, subsistencia y asentamiento en el curso medio del Río Quequén Grande: un enfoque arqueológico. Tesis Doctoral inédita. Facultad de Ciencias Naturales y Museo, UNLP. La Plata.

MARTÍNEZ, G. 2004. Resultados preliminares de las investigaciones arqueológicas realizadas en el curso inferior del río Colorado (partidos de Villarino y Patagones, provincia de Buenos Aires). En Aproximaciones Arqueológicas Pampeanas. Perspectivas Teóricas, Metodológicas, Analíticas y Casos de Estudio, editado por G. Martínez, M. Gutiérrez, R. Curtoni, M. Berón y P. Madrid, pp. 275-292. FACSO-UNICEN, Olavarría.

MARTÍNEZ, G. y M. GUTIÉRREZ. 2004. Tendencias en la explotación humana de la fauna durante el Pleistoceno final y Holoceno en la Región Pampeana (Argentina). En Zooarchaeology of South America, editado por G. Mengoni Goñalons, pp 81-98. British Archaeological Reports, International Series 1298. Archaeopress, Oxford.
MARTÍNEZ, G., G. FLENSBORG, P. BAYALA y R. LÓPEZ. 2007. Análisis de la composición anatómica, sexo y edad de dos entierros secundarios del sitio Paso Alsina 1 (Pdo. de Patagones, Pcia. de Buenos Aires). En Arqueología en las Pampas, editado por C. Bayón, I. González, N. Flegenheimer, A. Pupio y M. Frére, pp. 41-58. Sociedad Argentina de Antropología, Buenos Aires.

MAZZANTI, D. L. 2006. La constitución de territorios sociales durante el Holoceno tardío. El caso de las Sierras de Tandilla, Argentina. Relaciones de la Sociedad Argentina de Antropología 31: 277-300.

MEINDL, R. S. y C. O. LOVEJOY. 1985. Ectocranial suture closure: A revised method for the determination of skeletal age at death based on the lateral-anterior sutures. American Journal of Physical Anthropology 68: 57-66.

MC HUG, W. D. 1970. Dental Plaque. E \& S. Livingtone, Edinburgo.

MOLNAR, P. 2008. Dental wear and oral pathology: possible evidence and consequences of habitual use of teeth in a Swedish Neolithic sample. American Journal of Physical Anthropology 136: 423-431.

MENÉNDEZ, L., M. OSTERRIETH y F. OLIVA. 2009. A first phytolith approximation to diet study in the archaeological site Gascón 1, Pampean Region, República Argentina. Quaternary International 204 (2009), pp.84-94.

MORENO, F. 1874. Cementerios y paraderos prehistóricos de la Patagonia. Anales Científicos Argentinos 1:2-13.

OGDEN, A.R. 2008a. Advances in the paleopathology of teeth and jaws. En: Mays, S., \& Pinhaisi, R. (Eds.) Advance in Human paleopathology. John Wiley \& Sons, Chichester, pp. 283-307.

OGDEN, A.R. 2008b. Periapical voids in human jaw bones. En: Smith, M. \& Brickley, M. (Eds.), Proceedings of the $7^{\text {th }}$ British Association of Biological Anthropology and Human Osteoarchaeology (BABAO) Meeting. British Anthropological Reports, International Series: 1743: 51-56.

OXENHAM, M., N. L. CUONG, y N. K. TUY. 2006. The oral health consequences of the adoption and intensification of agriculture in Southeast Asia. En Bioarchaeology of Southeast Asia, editado por M. Oxenham y N. Tayles, pp 263-289.Cambridge University Press, Cambridge.

PARK, A.W. 1991. The ancient origins of oral pathology. Journal of Nihon University School of Dentistri 33 (4): 211-237.

POLITIS, G. 1984. Investigaciones arqueológicas en el área Interserrana Bonaerense. Etnía 32, 7-52, Olavarría.

POLITIS, G. 2008. The pampas and campos of South America. En Handbook of South American Archaeology, editado por H. Silverman y W. Isbell, pp. 235-260. Springer, Berlín. 
POLITIS, G., G. BARRIENTOS y T. W. STAFFORD, JR. 2009. Revisiting Ameghino: $\mathrm{New}{ }^{14} \mathrm{C}$ dates from ancient human skeletons from the Argentine pampas. En Premiers Peuplements et Préhistoire du Continent Américain, editado por D. Vialou. Comité des Travaux Historiques et Scientifiques, Paris. En prensa.

STINE, S. 1994. Extreme and persistent drought in California and Patagonia during mediaeval time. Nature 369: 546-549.

WALKER, P. L. y J. ERLANDSON. 1986. Dental evidence of prehistoric dietary change on the Northern Channel Islands, California. American Antiquity 51: 375-383.
TURNER II, C.G. 1979. Dental Anthropological indications of agricultura among the Jomon people of central Japan. American Journal of Physical Anthropology, 51: 619-636.

WHITTAKER, D., S. GRIFFITHS, A. ROBSON, P. ROGERDAVIES, G. THOMAS y T. MOLLESON. 1990. Continuing tooth eruption and alveolar crest height in an eighteenth-century population from Spitalfields, East London, Archives of Oral Biology 35: 81-85. 